\title{
ESTUDO DA DEGRADAÇÃO TÉRMICA DA MADEIRA DE Eucalyptus ATRAVÉS DE TERMOGRAVIMETRIA E CALORIMETRIA ${ }^{1}$
}

Bárbara Luísa Corradi Pereira ${ }^{2}$, Angélica de Cássia Oliveira Carneiro ${ }^{3}$, Ana Márcia Macedo Ladeira Carvalho $^{3}$, Paulo Fernando Trugilho ${ }^{4}$, Isabel Cristina Nogueira Alves Melo ${ }^{5}$ e Aylson Costa Oliveira ${ }^{2}$

\begin{abstract}
RESUMO - O objetivo deste trabalho foi avaliar a degradação térmica da madeira de diferentes clones de Eucalyptus, por meio de análises termogravimétrica (TG/DTG) e de calorimetria exploratória diferencial (DSC), estabelecendo-se suas relações com a produção de carvão vegetal. Foram utilizados seis clones de Eucalyptus spp. aos 7,5 anos de idade, provenientes de um teste clonal pertencente a uma empresa florestal. Foram realizadas as análises TG/DTG e DSC, sob atmosfera de gás nitrogênio. As curvas TG/DTG mostraram três faixas de degradação térmica, atribuídas à secagem da madeira e à degradação de hemiceluloses e celulose. Não foi detectada faixa específica de degradação de lignina. As curvas DSC evidenciaram um pico endotérmico entre as temperaturas de 50 e $100^{\circ} \mathrm{C}$, o que pode ser atribuído à perda de água da madeira. A liberação de calor iniciou-se em temperaturas acima de $275^{\circ} \mathrm{C}$, para todos os clones avaliados, sendo observados dois picos exotérmicos. As análises térmicas da madeira (TG/DTG e DSC) não apresentaram variações expressivas entre os clones. Conclui-se que, através das técnicas estudadas, é possível identificar as principais fases das reações de liberação ou absorção de energia e perda de massa, que estão diretamente ligadas à qualidade do carvão vegetal.
\end{abstract}

Palavras-chave: Pirólise; Componentes químicos; Carvão vegetal.

\section{STUDY OF THERMAL DEGRADATION OF Eucalyptus WOOD BY THERMOGRAVIMETRY AND CALORIMETRY}

\begin{abstract}
The objective of this study was to evaluate the wood thermal degradation of different Eucalyptus clones by thermogravimetric analysis (TG/DTG) and differential scanning calorimetry (DSC) by establishing their relationships with charcoal production. Six clones of Eucalyptus spp. were evaluated. Such clones were from a clonal test of a forest company. The analyses of TG/DTG and DSC were carried out in nitrogen atmosphere. The TG/DTG curves showed three bands of thermal degradation attributed to the drying of wood, cellulose and hemicelluloses degradation. A specific range of lignin degradation was not detected. DSC curves showed an endothermic peak between 50 and $100^{\circ} \mathrm{C}$, which can be attributed to water loss. The heat release started at temperatures above $275^{\circ} \mathrm{C}$, for all clones, in which two exothermic peaks were found. The thermal analysis of wood (TG / DTG and DSC) showed no significant variations among the clones. It can be concluded that by using the studied techniques, it is possible to identify the major stages of release or absorption of energy, and weight loss, which are directly related to the quality of charcoal.
\end{abstract}

Keywords: Pyrolysis; Chemical components; Charcoal.

\footnotetext{
${ }^{1}$ Recebido em 26.09.2012 aceito para publicação em 07.05.2013.

${ }^{2}$ Programa de Pós-Graduação em Ciência Florestal pela Universidade Federal de Viçosa, UFV, Brasil. E-mail: <babicorradi@gmail.com> e<aylsoncosta@gmail.com>.

${ }^{3}$ Departamento de Engenharia Florestal da Universidade Federal de Viçosa, UFV, Brasil. E-mail: <cassiacarneiro1@ gmail.com> e<ana.marcia@ufv.br>.

${ }^{4}$ Universidade Federal de Lavras, Laboratório de Tecnologia da Madeira. E-mail: <trugilho@ufla.br>.

${ }^{5}$ Universidade Federal Rural do Rio de Janeiro, Instituto de Florestas, UFRRJ, Brasil. E-mail: <bebelcrist@ yahoo.com.br>.
} 


\section{INTRODUÇÃO}

A pirólise da madeira é um processo físico-químico em que esta é aquecida a temperaturas até $450^{\circ} \mathrm{C} \mathrm{em}$ atmosfera não oxidante e tem como produto um resíduo sólido rico em carbono, o carvão vegetal e uma fração volátil que consiste em gases não condensáveis e vapores orgânicos condensáveis (ANTAL JR., 2003). Trata-se de um processo extremamente complexo e geralmente é constituída por uma série de reações químicas, acompanhadas por processos de transferência de calor e massa (YANG et al., 2007). A pirólise de qualquer biomassa, entre elas a madeira, pode ser considerada como a sobreposição da decomposição dos seus três componentes principais: hemiceluloses, celulose e ligninas, além da perda de água do material.

A celulose é o principal constituinte químico da madeira, constituindo de 40 a $45 \%$ da matéria seca da maioria das madeiras, sendo um polissacarídeo formado exclusivamente por unidades de $\beta$-D-anidroglicopiranose, unidas por ligações glicosídicas do tipo $\beta(1-4)$ (SJÖSTRÖM, 1993; ROWELL et al., 2005). Esse polímero se decompõe em temperaturas compreendidas entre 315 e $400{ }^{\circ} \mathrm{C}$ (YANG et al., 2007). Já as hemiceluloses representam, em média, 20 a $30 \%$ da massa seca da madeira (SJÖSTRÖM, 1993). São polímeros geralmente amorfos, de baixo peso molecular, constituídos de uma cadeia central de unidades repetitivas acrescida de cadeias laterais e ramificações (SJÖSTRÖM, 1993; ROWELL et al., 2005). Sua degradação térmica ocorre entre 190 e $360^{\circ} \mathrm{C}$, de acordo com Shen et al. (2010).

Por fim, as ligninas são macromoléculas tridimensionais, amorfas e ramificadas e apresentam o fenilpropano como unidade básica, unidos por ligações do tipo éter (C-O-C) e carbono-carbono (C-C) (ROWELL et al., 2005). Sua decomposição térmica ocorre em ampla faixa de temperatura, a partir de $100^{\circ} \mathrm{C}$, até temperaturas próximas a $900^{\circ} \mathrm{C}$, podendo ser considerada um conjunto de várias reações paralelas (MÜLLER-HAGEDORN et al., 2003; YANG et al., 2007).

Assim, a melhor compreensão do comportamento da madeira durante a pirólise é decisiva para a seleção de clones, otimização dos processos e melhoria da qualidade do carvão vegetal. Considerando a grande variedade de espécies e clones de Eucalyptus cultivados no Brasil, torna-se necessária a busca por novas informações sobre as propriedades da madeira, para que a seleção de materiais genéticos superiores para produção de carvão vegetal seja bem-sucedida.
Diante do exposto, o objetivo deste trabalho foi avaliar a degradação térmica da madeira de diferentes clones de Eucalyptus por meio das análises termogravimétrica (TG/DTG) e de calorimetria exploratória diferencial (DSC), estabelecendo suas relações com a produção de carvão vegetal.

\section{MATERIAL E MÉTODOS}

\subsection{Material biológico}

Neste trabalho foram utilizados seis clones de Eucalyptus spp. (Tabela 1) aos 7,5 anos de idade, cultivados em espaçamento $3,8 \times 2,4 \mathrm{~m}$, provenientes de um teste clonal em linhas de cinco plantas com quatro repetições, pertencente a uma empresa florestal, localizada no Município de Lassance, Minas Gerais.

Foram selecionadas três árvores de diâmetro médio para cada um dos seis clones, totalizando 18 árvores (amostras). A seleção foi feita excluindo-se as árvores que apresentavam defeitos visíveis a olho nu e também aquelas que estavam nas bordas do plantio.

O experimento foi conduzido no Laboratório de Painéis e Energia da Madeira - LAPEM, no Laboratório de Propriedades da Madeira do Departamento de Engenharia Florestal da Universidade Federal de Viçosa (UFV) e Laboratório de Biomateriais do Departamento de Ciências Florestais da Universidade Federal de Lavras (UFLA).

\subsection{Preparo de amostras}

De cada árvore foram retirados seis discos de $10 \mathrm{~cm}$ de espessura, correspondentes a $0 \%, 25 \%, 50 \%$, $75 \%$ e $100 \%$ da altura comercial do tronco até o diâmetro mínimo de $7 \mathrm{~cm}$.

As amostras de madeira foram transformadas em serragem, utilizando-se um moinho de laboratório tipo Wiley, de acordo com a norma TAPPI 257 om-52 (TAPPI, 2001). Foram feitas amostras compostas de cada árvore e utilizada a fração de granulometria classificada entre as peneiras de 200 e 270 mesh. A umidade média das amostras era de $6,47 \%$.

\subsection{Análise termogravimétrica (TG/DTG)}

Para análise termogravimétrica da madeira, utilizou-se o aparelho DTG-60H, Shimadzu. As análises foram realizadas sob atmosfera de gás nitrogênio, a uma vazão constante de $50 \mathrm{ml} \cdot \mathrm{min}^{-1}$, utilizando-se aproximadamente $2 \mathrm{mg}$, em cápsula de alumina aberta. As curvas 
Tabela 1 - Informações gerais sobre os diferentes materiais genéticos utilizados no estudo e composição química da madeira. Table 1 - General information about the different genetic materials used in the study and chemical composition of wood.

\begin{tabular}{|c|c|c|c|c|c|}
\hline \multicolumn{6}{|c|}{ Informações gerais } \\
\hline Clone & \multicolumn{2}{|c|}{ Material genético } & \multicolumn{2}{|c|}{ Procedência } & IMA \\
\hline 1 & \multicolumn{2}{|c|}{ Eucalyptus camaldulensis } & \multicolumn{2}{|c|}{ Plantar S.A./Curvelo,MG } & 39,6 \\
\hline 2 & \multicolumn{2}{|c|}{ Eucalyptus urophylla (híbrido) } & \multicolumn{2}{|c|}{ Gerdau S.A./Três Marias, MG } & 32,4 \\
\hline 3 & \multicolumn{2}{|c|}{ Eucalyptus grandis (híbrido) } & \multicolumn{2}{|c|}{ Suzano/Teixeira de Freitas, BA } & 40,7 \\
\hline 4 & \multicolumn{2}{|c|}{ Eucalyptus urophylla (híbrido) } & \multicolumn{2}{|c|}{ Gerdau S.A./Três Marias, MG } & 35,9 \\
\hline 5 & \multicolumn{2}{|c|}{ Eucalyptus urophylla } & \multicolumn{2}{|c|}{ V \& M Florestal/João Pinheiro, MG } & 31,5 \\
\hline 6 & \multicolumn{2}{|c|}{ Eucalyptus camaldulensis } & \multicolumn{2}{|c|}{ Gerdau S.A./Três Marias, MG } & 40,3 \\
\hline \multicolumn{6}{|c|}{ Composição química } \\
\hline Clone & Celulose* $(\%)$ & Hemiceluloses* $(\%)$ & Lignina* $(\%)$ & Extrativos $(\%)$ & Cinzas $(\%)$ \\
\hline 1 & 47,6 & 22,1 & 30,3 & 4,3 & 0,16 \\
\hline 2 & 46,1 & 22,5 & 31,4 & 5,0 & 0,14 \\
\hline 3 & 48,2 & 22,0 & 29,8 & 4,1 & 0,12 \\
\hline 4 & 48,8 & 22,4 & 28,8 & 4,7 & 0,10 \\
\hline 5 & 48,4 & 21,9 & 29,7 & 4,8 & 0,11 \\
\hline 6 & 47,2 & 22,4 & 30,4 & 3,1 & 0,18 \\
\hline
\end{tabular}

IMA = incremento médio anual volumétrico com casca $\left(\mathrm{m}^{3} \cdot \mathrm{ha}^{-1} \cdot \mathrm{ano}^{-1}\right) ; \mathrm{e} *$ Madeira livre de extrativos

termogravimétricas foram obtidas a partir de $50{ }^{\circ} \mathrm{C}$ até a temperatura máxima de $450^{\circ} \mathrm{C}$, com taxa de aquecimento de $10^{\circ} \mathrm{C} \cdot \mathrm{min}^{-1}$.

A curva termogravimétrica (TG) foi obtida para avaliar a perda de massa em função da temperatura e da curva da derivada primeira da perda de massa (DTG).

A partir das curvas TG, procederam-se aos cálculos de perda de massa nos seguintes intervalos de temperatura: temperatura $50-100{ }^{\circ} \mathrm{C}, 100-150{ }^{\circ} \mathrm{C}, 150$ $200{ }^{\circ} \mathrm{C}, 200-250{ }^{\circ} \mathrm{C}, 250-300{ }^{\circ} \mathrm{C}, 300-350{ }^{\circ} \mathrm{C}, 350-400$ ${ }^{\circ} \mathrm{Ce} 400-450{ }^{\circ} \mathrm{C}$. Calculou-se, também, a massa residual na temperatura de $450{ }^{\circ} \mathrm{C}$, considerando a massa absolutamente seca, na temperatura de $100{ }^{\circ} \mathrm{C}$.

\subsection{Calorimetria diferencial exploratória (DSC)}

Para a calorimetria exploratória diferencial (DSC), foi utilizado o equipamento DSC-60A, Shimadzu. As curvas DSC foram obtidas em atmosfera de nitrogênio com vazão constante de $50 \mathrm{ml} \cdot \mathrm{min}^{-1}$, utilizando-se uma cápsula de alumínio prensada. Foram usados aproximadamente $2 \mathrm{mg}$ de serragem, e procedeu-se à análise da temperatura inicial de $50^{\circ} \mathrm{C}$ até a temperatura máxima de $450^{\circ} \mathrm{C}$, com taxa de aquecimento de $10^{\circ} \mathrm{C}$. min

\subsection{Análise estatística}

O experimento foi instalado segundo um delineamento inteiramente casualizado, com seis tratamentos (clones), em três repetições (árvores), totalizando 18 unidades amostrais.

Os dados foram submetidos aos testes de Lilliefors, para testar a normalidade, e de Cochran, para testar a homogeneidade das variâncias. Em seguida, os resultados foram submetidos à análise de variância (ANOVA), para verificação das diferenças entre os clones avaliados. Quando estabelecidas as diferenças significativas entre eles, aplicou-se o teste de Tukey em nível de $95 \%$ de significância.

As análises estatísticas foram realizadas com o auxílio do software STATISTICA 8.0 (2007).

\section{RESULTADOS}

\subsection{Análise termogravimétrica (TG/DTG)}

As curvas termogravimétricas (TG) representam a perda de massa, em porcentagem, em função da temperatura, enquanto as curvas DTG correspondem à derivada primeira das curvas TG e apresentam a variação da massa em relação ao tempo, registradas em função da temperatura.

Na Figura 1 estão representadas as curvas termogravimétricas (TG/DTG) da madeira, para os seis clones de Eucalyptus, a partir da curva intermediária obtida para cada clone, na faixa de temperatura de 50 a $450{ }^{\circ} \mathrm{C}$, temperatura máxima recomendada para a produção de carvão vegetal.

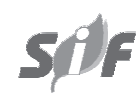

Revista Árvore, Viçosa-MG, v.37, n.3, p.567-576, 2013 

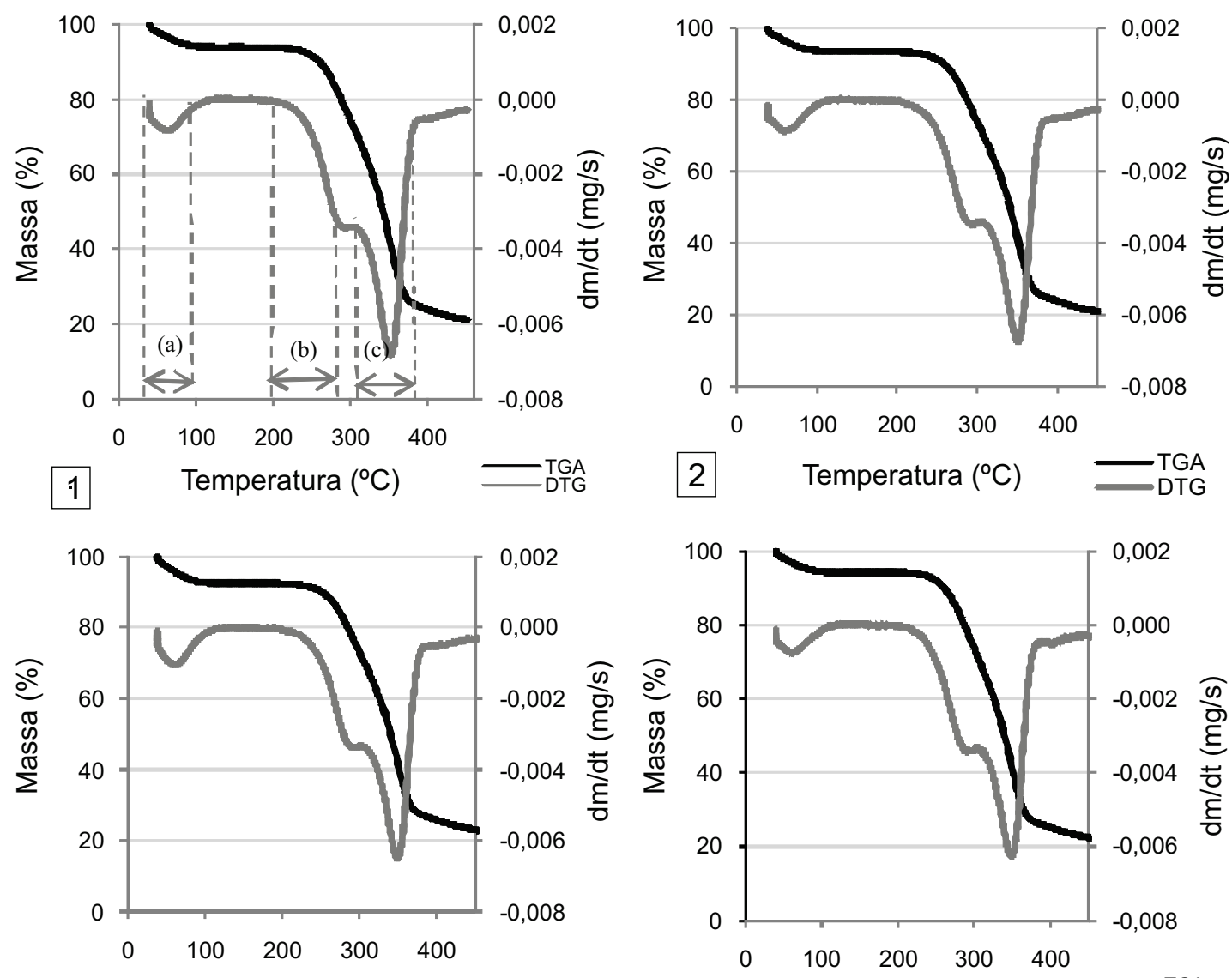

\section{3}
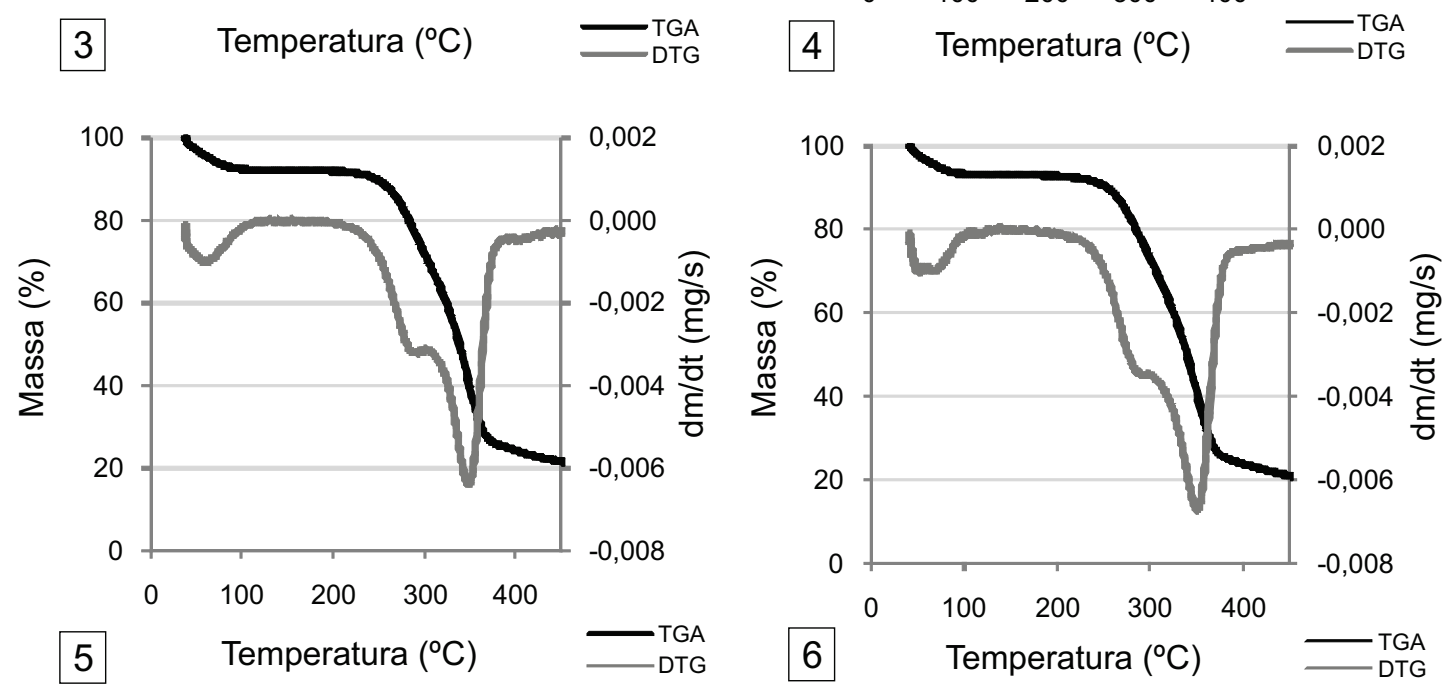

Figura 1 - Curvas TG/DTG da madeira de Eucalyptus spp. para os clones 1, 2, 3, 45 e 6. Atmosfera de nitrogênio e taxa de aquecimento de $10^{\circ} \mathrm{C} / \mathrm{min}$. (a) Secagem da madeira, (b) Degradação térmica de hemiceluloses e (c) Degradação térmica de celulose.

Figure 1-TG/DTG curves of Eucalyptus spp. wood, for clones 1, 2, 3, 4, 5 and 6. Nitrogen atmosphere and heating rate of $10^{\circ} \mathrm{C} / \mathrm{min}$. (a) wood drying, (b) thermal degradation of hemicelluloses and (c) thermal degradation of cellulose

Revista Árvore, Viçosa-MG, v.37, n.3, p.567-576, 2013 
Observa-se, na Figura 1, que os perfis de degradação térmica da madeira dos diferentes clones apresentaram-se semelhantes, ocorrendo pequenas diferenciações nas temperaturas correspondentes aos picos máximos de degradação, relacionados à degradação das hemiceluloses e celulose, principalmente. Observa-se, também, que os picos máximos de degradação das hemiceluloses ocorreram em temperaturas entre 278 e $280^{\circ} \mathrm{C}$ e da celulose, entre 342 e $347^{\circ} \mathrm{C}$.

As curvas TG/DTG indicam três faixas de degradação térmica, que estão destacadas na Figura 1.1, sendo a primeira atribuída à secagem da madeira e as outras duas, à degradação de hemiceluloses e celulose, devendo-se ressaltar que cada um se degrada de maneira distinta em diferentes faixas de temperatura. Não foi detectada faixa específica de degradação de lignina, devido ao fato de esta ocorrer em ampla faixa de temperatura (YANG et al., 2007), além de a sua temperatura final de degradação ser superior à temperatura final avaliada $\left(450^{\circ} \mathrm{C}\right)$, havendo sobreposição das faixas.

A segunda faixa de temperatura que corresponde, principalmente, à degradação térmica das hemiceluloses e de menores proporções de celulose e lignina teve início em 201,29; 203,24; 207,13;214,91; 203,24; e $201,29^{\circ} \mathrm{C}$, respectivamente para os clones $1,2,3,4$, 5 e 6 . Seu término ocorreu nas temperaturas de 289,50; 293,$31 ; 293,32 ; 295,22 ; 293,31$; e $287,60{ }^{\circ} \mathrm{C}$, respectivamente para os clones $1,2,3,4,5$ e 6 .

A terceira faixa de degradação térmica iniciou-se em seguida e se estendeu até 387,20; 389,07; 385,34; 389,$07 ; 390,93$; e 400,21 ${ }^{\circ} \mathrm{C}$ para os clones $1,2,3,4$, 5 e 6 , respectivamente, sendo esta faixa de temperatura que corresponde, principalmente, à degradação da celulose. As taxas máximas de perda de massa foram alcançadas em temperaturas próximas a $350{ }^{\circ} \mathrm{C}$ para todos os clones, assim como outras espécies, a exemplo do estudo realizado por Vidaurre et al. (2012), que avaliaram a madeira de Schizolobium amazonicum.

A partir de temperaturas próximas a $400^{\circ} \mathrm{C}$, verifica-se que a degradação térmica da madeira se tornou menor, correspondendo, principalmente, à degradação de lignina. Nessa temperatura, a celulose e as hemiceluloses, componentes químicos em maior proporção na madeira, já foram degradadas.

Na Tabela 2, apresentam-se as médias das perdas de massa obtidas para cada clone de Eucalyptus, em função de faixas de temperatura de 50 a $450{ }^{\circ} \mathrm{C}$, com intervalos de $50^{\circ} \mathrm{C}$, e a massa residual no fim do processo.

Não foram verificadas diferenças significativas a 95\% de significância para a perda de massa nessas faixas de temperatura, apesar das diferenças na composição química da madeira dos clones avaliados. Também não foram observadas diferenças significativas para a massa residual, quando considerada a massa seca de madeira.

\subsection{Calorimetria diferencial exploratória (DSC)}

Na Figura 2 estão representadas as curvas DSC da madeira para os seis clones avaliados, a partir da curva intermediária obtida para cada clone. ADSC fornece a informação se a reação é caracterizada por liberação de calor (exotérmica) ou absorção de calor (endotérmica), além de fornecer a quantidade de calor envolvido na reação.

De acordo com Oliveira (2009), os dados de DSC devem ser analisados sempre com cautela, já que eles mostram o efeito total das reações que incidem diretamente sobre a madeira, denominadas reações primárias, e aquelas que incidem na decomposição dos produtos intermediários, como vapores orgânicos e levoglucosana, denominadas reações secundárias, sendo essas reações complexas, consecutivas e, ou, simultâneas.

Na Tabela 3 estão as temperaturas relacionadas ao início do processo exotérmico e aos picos máximos das curvas DSC da madeira dos diferentes clones de Eucalyptus spp.

\section{DISCUSSÃO}

\subsection{Análise termogravimétrica (TG/DTG)}

Na faixa inicial de degradação, que corresponde à fase de secagem da madeira, observou-se perda de massa média de $6,47 \%$. Nessa fase ocorre perda da água de adesão presente na parede celular e que necessita de energia para ser retirada da madeira, sendo esta fase, portanto, endotérmica.

Constatou-se que na faixa de temperatura compreendida entre 100 e $200{ }^{\circ} \mathrm{C}$ a perda de massa foi mínima $(0,36 \%$, em média). De acordo com Randriamantena et al. (2009), essa é a chamada zona de estabilidade térmica da madeira, que é limitada pela temperatura inicial de degradação térmica dos principais

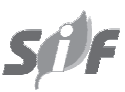

Revista Árvore, Viçosa-MG, v.37, n.3, p.567-576, 2013 
Tabela 2 - Valores médios da perda de massa dos clones de Eucalyptus em função das faixas de temperatura e massa residual, em porcentagem.

Table 2 -Average values of Eucalyptus clones wood weight loss depending temperature ranges, and residual mass, in percentag.

\begin{tabular}{cccccccccc}
\hline \multirow{2}{*}{ Clones } & \multicolumn{7}{c}{ Faixas de temperatura $\left({ }^{\circ} \mathrm{C}\right)$} \\
\cline { 2 - 8 } & $50-100$ & $100-150$ & $150-200$ & $200-250$ & $250-300$ & $300-350$ & $350-400$ & $400-450$ \\
\hline 1 & 6,22 & 0,22 & 0,09 & 2,19 & 17,14 & 31,62 & 15,48 & 2,81 \\
2 & 5,56 & 0,22 & 0,11 & 2,75 & 17,96 & 32,62 & 15,86 & 2,86 \\
3 & 6,41 & 0,22 & 0,08 & 2,23 & 17,39 & 32,01 & 15,18 & 3,00 & 25,11 \\
4 & 6,64 & 0,18 & 0,12 & 2,13 & 17,13 & 32,26 & 17,89 & 2,84 & 28,55 \\
5 & 7,26 & 0,34 & 0,14 & 2,16 & 17,76 & 32,35 & 15,07 & 2,87 \\
6 & 6,75 & 0,28 & 0,17 & 2,38 & 17,14 & 31,71 & 15,54 & 2,37 \\
Média & 6,47 & 0,24 & 0,12 & 2,31 & 17,42 & 32,09 & 15,83 & 2,79 \\
\hline
\end{tabular}

*Massa residual, considerando-se a massa de madeira absolutamente seca (a.s.).

componentes da madeira. Vale ressaltar que os componentes da madeira são termicamente estáveis nessa faixa de temperatura, desde que não sejam expostos ao calor por períodos prolongados (RAAD et al., 2006). Em estudos termogravimétricos da madeira, Santos et al. (2012) não observaram perda de massa nessa faixa de temperatura, ao estudarem quatro clones de Eucalyptus spp. aos 7 anos, e Castro (2011) verificou perda de massa média de $0,53 \%$ para três clones de Eucalyptus spp., também aos 7 anos.

De 250 a $300^{\circ} \mathrm{C}$, verifica-se que a perda de massa média foi de $17,42 \%$; pode-se inferir que a maior parte dessa massa perdida é atribuída à degradação das hemiceluloses. Entre as temperaturas de 200 e $300{ }^{\circ} \mathrm{C}$, Santos et al. (2012) observaram perdas de massa entre 16 e 19\%, e Castro (2011) constatou, em média, 19,6\% de perda de massa, ambos para clones de Eucalyptus spp. aos 7 anos de idade, valores esses correspondentes aos verificados neste estudo.

Entre as faixas de temperatura de 300 a $350^{\circ} \mathrm{C}$ e 350 a $400{ }^{\circ} \mathrm{C}$ ocorreram as maiores perdas de massa, 32,09 e $15,83 \%$, em média, totalizando $47,93 \%$. Liao (2003) sugeriu que o pico de perda de massa para a celulose é posterior à degradação térmica das hemiceluloses, devido à maior quantidade de energia necessária para a despolimerização da cadeia de celulose e a quebra de seus monômeros.

Se for considerada a porcentagem total de celulose presente na madeira dos clones deste trabalho, em torno de $48 \%$, pode-se dizer que a celulose foi, quase em sua totalidade, degradada nessa faixa de temperatura, mesmo considerando que há degradação, em menores proporções, dos demais constituintes químicos da madeira, lignina e hemiceluloses. Santos et al. (2012) e Castro (2011) verificaram perdas de massa iguais a 46,4 e $50 \%$, respectivamente, entre as temperaturas de 300 a $400^{\circ} \mathrm{C}$. Considerando-se a utilização da madeira para a produção de carvão vegetal, o fato explicitado anteriormente evidencia a baixa contribuição da celulose para o rendimento em carvão vegetal, ainda que este seja o componente presente em maior porcentagem na madeira. Alguns autores mencionaram que a massa residual de celulose em temperatura de $450{ }^{\circ} \mathrm{C}$ varia entre 5 e 10\% (DI BLASI, 1998; YANG et al., 2007; SHEN et al., 2010).

A ausência de um pico de degradação referente à lignina é devida, provavelmente, ao fato de a sua decomposição térmica ocorrer em ampla faixa de temperatura, ressaltando-se que apenas pequena fração se decompõe em temperaturas inferiores a $450^{\circ} \mathrm{C}$, cerca de $40 \%$ de sua massa, conforme citado por Várhegyi et al. (1997), Müller-Hagedorn et al. (2003) e Yang et al. (2007). Portanto, o rendimento em carvão vegetal está intimamente relacionado à composição química da madeira, no que se diz respeito a elevados teores de lignina, aliado às variáveis do processo de produção. Vale salientar que a temperatura de $450^{\circ} \mathrm{C}$ é a máxima recomendada para a produção de carvão vegetal, sendo nessa temperatura possível atrelar maiores rendimentos à qualidade do carvão vegetal requerida para o uso siderúrgico. A partir dessa temperatura, a degradação da lignina começa a ser mais intensa, o que prejudica o rendimento e as propriedades do carvão vegetal.

Ao considerar a massa residual obtida para cada clone, quando foi considerada a madeira absolutamente 

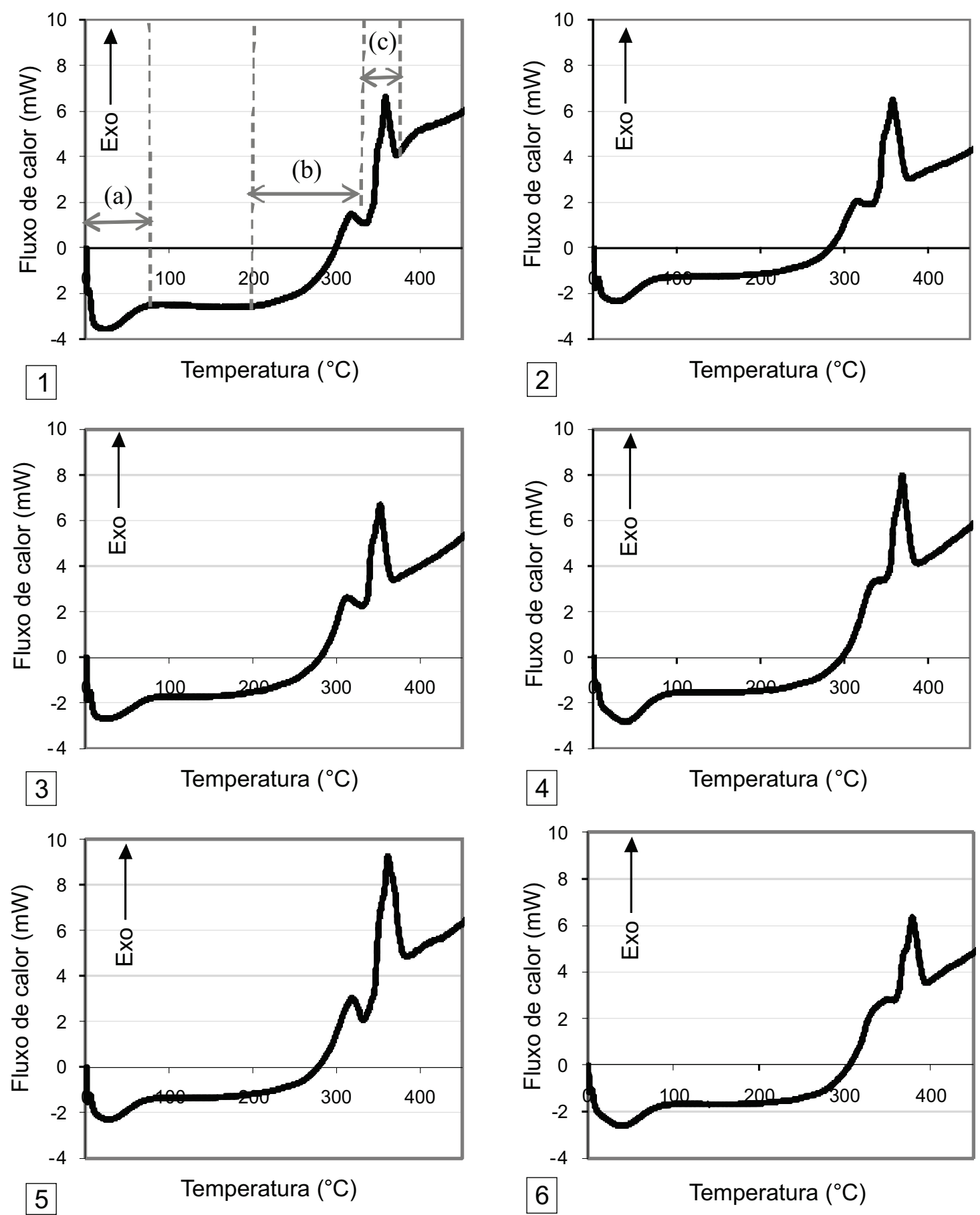

Figura 2 - Curvas DSC da madeira de Eucalyptus para os clones 1, 2, 3, 4, 5 e 6. Atmosfera de nitrogênio e taxa de aquecimento de $10^{\circ} \mathrm{C} / \mathrm{min}$. (a) Secagem da madeira, (b) Degradação térmica de hemiceluloses e (c) Degradação térmica de celulose.

Figure 2-DSC curves of Eucalyptus spp. wood, for clones 1, 2, 3, 4, 5 and 6. Nitrogen atmosphere and heating rate of $10^{\circ} \mathrm{C} / \mathrm{min}$. (a) wood drying, (b) thermal degradation of hemicelluloses and (c) thermal degradation of cellulose. 
Tabela 3 - Temperaturas relacionadas com o início do processo exotérmico e com os picos máximos das curvas DSC da madeira de Eucalyptus.

Table 3 -Temperatures related to the beginning of exothermic process and maximum peak of Eucalyptus wood DSC curves.

\begin{tabular}{cccc}
\hline \multirow{2}{*}{ Clones } & & Temperatura $\left({ }^{\circ} \mathrm{C}\right)$ \\
\cline { 2 - 3 } & Início fase exotérmica & Máxima ${ }^{\circ}$ Pico exotérmico & Máxima $2^{\circ}$ Pico exotérmico \\
\hline 1 & 302,74 & 328,33 & 369,47 \\
2 & 293,61 & 327,88 & 369,90 \\
3 & 283,99 & 317,33 & 357,53 \\
4 & 298,38 & 341,30 & 36,14 \\
6 & 276,05 & 324,51 & 38,29 \\
\hline
\end{tabular}

seca, verificou-se que não houve diferenças significativas entre os clones, o que indica que a resistência à degradação térmica dos diferentes materiais genéticos avaliados é similar.

As massas residuais médias obtidas variaram de 25,4 a $27,6 \%$ para os clones 6 e 3 , respectivamente. Esses valores são inferiores aos comumente obtidos para o rendimento gravimétrico em carvão vegetal obtido em mufla, entre 30 e 36\% (BOTREL et al., 2007; OLIVEIRA et al., 2010; OLIVEIRA et al., 2012). Tal fato é devido, principalmente, à maior área superficial da madeira nas análises termogravimétricas, uma vez que as análises são realizadas com madeira moída e classificada em peneira de 270 mesh e também à taxa de aquecimento utilizada, maior na análise termogravimétrica em relação à carbonização em mufla.

Verificou-se que os clones avaliados neste estudo são mais resistentes à degradação térmica quando comparados aos clones de Eucalyptus spp., aos 7 anos, avaliados também por termogravimetria por Santos et al. (2012), e menos resistentes à degradação térmica quando comparados aos clones de Eucalyptus avaliados por Castro (2011), com a mesma idade. As autoras encontraram massa sólida residual média de 14,75 e $30,2 \%$, respectivamente.

\subsection{Calorimetria diferencial exploratória (DSC)}

As curvas DSC indicaram um pico endotérmico entre as temperaturas de 50 e $100^{\circ} \mathrm{C}$, que pode ser atribuído à perda de água da madeira, pois se faz necessária a energia para a sua secagem. De acordo com Oliveira (2003), inicialmente a madeira absorve energia necessária à secagem e ruptura das ligações químicas de seus componentes estruturais, passando a liberar energia assim que os produtos de degradação térmica vão sendo formados. Segundo Leroy et al. (2010), dióxido de carbono e traços de compostos orgânicos são liberados entre as temperaturas de 100 a $200{ }^{\circ} \mathrm{C}$.

Observou-se que a liberação de calor inicia-se em temperaturas acima de $275^{\circ} \mathrm{C}$, para todos os clones avaliados, evidenciando que os componentes químicos da madeira começam a se decompor a partir dessa temperatura, conforme mencionado na análise termogravimétrica (Figura 2). Entre os clones avaliados, a madeira do clone 5 foi a que apresentou o início do processo exotérmico em menor temperatura $\left(276,05^{\circ} \mathrm{C}\right)$, enquanto para a madeira do clone 6 tal fato ocorreu em maior temperatura $\left(306,17^{\circ} \mathrm{C}\right)$, em relação aos demais clones (Tabela 3).

Verifica-se a presença de dois picos exotérmicos para a decomposição térmica da madeira dos clones de Eucalyptus, o que está em conformidade com o os trabalhos realizados por Tsujiyama e Miyamori (2000) e Leroy et al. (2010). De acordo com esses mesmos autores, esses picos são atribuídos à entalpia de formação dos produtos gasosos liberados durante a pirólise.

O primeiro pico exotérmico foi verificado entre as temperaturas de $317,33^{\circ} \mathrm{C}$ (clone 3 ) a $341^{\circ} \mathrm{C}$ (clone 4) e pode ser atribuído, principalmente, à degradação térmica das hemiceluloses. Contudo, essas temperaturas são relativamente superiores às encontradas em trabalhos em que a degradação das hemiceluloses foi avaliada (YANG et al., 2007; SHEN et al., 2010), o que pode ser explicado, provavelmente, pela degradação simultânea de outros componentes da madeira, principalmente lignina. Porém, Leroy et al. (2010), ao estudarem a decomposição térmica de holoceluloses da madeira 
de quatro espécies nativas de Córsega, encontraram picos exotérmicos próximos às temperaturas citadas, atribuídos à decomposição das hemiceluloses.

Posteriormente a esse primeiro pico, pôde-se verificar queda no fluxo de calor em ambos os clones. Esse comportamento pode ser relacionado à pirólise da celulose, que é endotérmica, devido ao elevado gasto de energia nas reações de despolimerização (YANG et al., 2007; HAYKIRI-ACMA et al., 2010).

O segundo pico exotérmico ocorreu em temperaturas que variaram de $357,53^{\circ} \mathrm{C}$ (clone 3) a $381,58^{\circ} \mathrm{C}$ (clone 6). Oliveira (2009) citou que esse pico se deve à degradação dos produtos originários durante a decomposição térmica da celulose. Ou seja, a degradação da celulose é endotérmica, porém tal degradação origina produtos que sofrem degradação secundária, que é exotérmica. Observou-se que o fluxo de calor liberado no segundo pico foi maior em relação ao primeiro, uma vez que, quanto maior a formação de produtos gasosos, maior também a energia liberada (OLIVEIRA, 2003), e os picos referentes à madeira dos clones 4 e 5 apresentaram maiores amplitudes em razão, provavelmente, dos seus maiores teores de celulose em relação aos dos demais clones.

A partir de aproximadamente $380^{\circ} \mathrm{C}$ até $450{ }^{\circ} \mathrm{C}$, o fluxo de calor, independentemente do clone, tornou-se linear e crescente, provavelmente devido à degradação térmica da lignina, cuja liberação de calor se torna mais intensa a partir de temperaturas próximas a $400^{\circ} \mathrm{C}$ (YANG et al., 2007; SHEN et al., 2010).

\section{CONCLUSÃO}

As análises térmicas da madeira (TG/DTG e DSC) não apresentaram variações expressivas entre os clones, sendo possível distinguir duas etapas de degradação, das hemiceluloses e celulose, além da secagem da madeira.

Através das técnicas estudadas, é possível identificar as principais fases das reações de liberação ou absorção de energia e perda de massa, que estão diretamente ligadas à qualidade do carvão vegetal.

A termogravimetria e a calorimetria podem subsidiar o controle do processo de carbonização em fornos através da associação da temperatura interna destes às fases de degradação dos componentes da madeira. Assim, podem-se estabelecer períodos em que o forno deve permanencer em cada faixa de temperatura, o que pode contribuir para o aumento do rendimento de carvão vegetal.

\section{AGRADECIMENTOS}

À Fundação de Amparo à Pesquisa do Estado de Minas Gerais - FAPEMIG, ao Conselho Nacional de Pesquisa e Desenvolvimento - CNPq e ao Grupo de Pesquisa de Carvão Vegetal - G6, pelo apoio finaceiro.

\section{REFERÊNCIAS}

ANTAL JR., M. The art, science, and technology of charcoal production. Industrial Engineering Chemistry Research, v.42, p.1619-1640, 2003.

BOTREL, M. C. G. et al.Melhoramento genético das propriedades docarvão vegetal de

Eucalyptus. Revista Árvore, v.31,n.3, p.391398, 2007.

CAStro, A. F. N. M. Efeito da idade e de materiais genéticos de Eucalyptus sp. na madeira e carvão vegetal. 2011. $97 \mathrm{f}$.

Dissertação (Mestrado em Ciência Florestal) Universidade Federal de Viçosa, Viçosa, MG, 2011.

DI BLASI, C. D. Comparison of semi-global mechanisms for primary pyrolysisof lignocellulosic fuels. Journal of Analytical and Applied Pyrolysis, v.47, n.1, p.43-64, 1998.

HAYKIRI-ACMA, H.; YAMAN, S.; KUCUKBAYRAK,S. Comparison of the thermal reactivities of isolated lignin and holocellulose during pyrolysis. Fuel Processing Technology, v.91, p.759-764, 2010.

KIM, U. J.; EOM, S. H.; WADA, M. Thermal decomposition of native cellulose: influence on crystallite size. Polymer Degradation and Stability, v.95, p.778-781, 2010.

LEROY, V.; LEONI, E.; CANCELLIERI, D. Thermal degradation of ligno-cellulosic fuels: biopolymers contribution.In: ELNASHAR, M.M (Ed). Biopolymers. Rijeka: Scyio, 2010. p.303-318.

LIAO, Y. F. Mechanism study of cellulose pyrolysis. Tese (PósDoutorado) ZheJiang University, HangZhou, China, 2003.

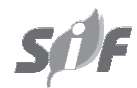

Revista Árvore, Viçosa-MG, v.37, n.3, p.567-576, 2013 
MÜLLER-HAGEDORN, M. et al. A comparative kinetic study on the pyrolysis of three different wood species. Journal of Analytical and Applied Pyrolysis, v.68, n.1, p.231-249, 2003.

OLIVEIRA, A. C. etal.Parâmetros de qualidade da madeira e do carvão vegetal de Eucalyptus pellita F. Muell. ScientiaForestalis, v.38, n.87, p.431-439, 2010.

OLIVEIRA, A. C. et al. Avaliação de diferentes níveis de desbaste nas propriedades da madeira e do carvão vegetal de Eucalyptus grandis x Eucalyptus urophylla. Floresta, v.42, n.1, p.59-68, 2012.

OLIVEIRA, E. Características anatômicas, químicas e térmicas da madeira de três espécies de maior ocorrência no semi-árido nordestino. 2003. 149f. Tese (Doutorado em Ciência Florestal) Universidade Federal de Viçosa, Viçosa, MG, 2003.

OLIVEIRA, R. M. Utilização de técnicas de caracterização de superficies de madeiras tratadas termicamente. 2009. 123f. Tese (Doutorado em Ciências/Física Aplicada) - Instituto de Física da Universidade de São Paulo, São Carlos, 2009.

POLETTO, M. et al. Thermal decomposition of wood: Influence of wood components and cellulose crystallite size. Bioresource

Technology,v.109, p.148-153, 2012.(Special Issue: Innovative Researches on Algal Biomass),

RAAD, T. J.; PINHEIRO, P. C. C.; YOSHIDA, M.I.Equação geral de mecanismos cinéticos da carbonização do Eucalyptus spp. Cerne, v.12, n.2, p.93-106, 2006.

RANDRIAMANANTENA, T. et al. Thermal behaviour of three woods of Madagascar by thermogravimetric analysis in inert atmosphere. In: HIGH-ENERGY PHYSICS INTERNATIONALCONFERENCE, 4., 2009, Madagascar. Anais... Madagascar: 2009.
ROWELL, R. M. et al. Cell wall chemistry. In: ROWELL, R.M. (Ed.). Handbook of wood chemistry and wood composites.Boca Raton: CRC Press, 2005. p.121-138.

SANTOS, R. C. et al. Análise termogravimétrica em clones de eucalipto como subsídiopara a produção de carvão vegetal. 2012. Cerne, v.18, n.1, p.143-151, 2012.

SHEN, R.; GU, S.; BRIDGWATER, A. V. The thermal performance of the polysaccharides extracted from hardwood: Cellulose and hemicelluloses. Carbohydrate Polymers, v.82, p. 39-45, 2010.

SJÖSTRÖM, E. Wood chemistry fundamentals and applications. London: Academic Press, 1993. 293p.

STATSOFT INC. Statistica data analysis system version 8.0. Tulsa: 2007.

TECHNICAL ASSOCIATION OF THE PULP AND PAPER INDUSTRY - TAPPI -. TAPPI test methods T $257 \mathrm{~cm}-85$ : sampling and preparing wood for analysis. In: TAPPI Standard Method. Atlanta: 2001.CD-ROM

TSUJIYAMA, S.; MIYAMORI, A. Assignment of DSC thermograms of wood and its components. Thermochimica Acta, v.351, p.177-181, 2000.

VÁRHEGYI, G. et al. Kinetic modeling of biomass pyrolysis. Journal of Analytical and Applied Pyrolysis, v.42, n.1, p.73-87, 1997.

VIDAURRE, G. B. et al. Propriedades energéticas da madeira e do carvão de paricá (Schizolobium amazonicum). Revista Árvore, v.36, n.2, p.365-371, 2012. 